\title{
Acoustic Radiation from an Automotive Gear Box
}

\author{
Rajendra Gunda and Sandeep Vijayakar
}

Advanced Numerical Solutions LLC

Copyright (C) 2007 SAE International

\begin{abstract}
The main goal of this paper is to extend the acoustic boundary element analysis to the high frequency regime. At higher frequencies, the surface boundary element mesh of the radiator must be sufficiently fine to capture the rapid spatial variations of the sound field. Traditional BEM implementations have had difficulty in dealing with large number of unknown variables and thus are limited to low frequency analysis of small bodies. In a typical boundary element analysis, computation of the coefficient matrix constitutes a major portion of the total analysis time and total memory usage.
\end{abstract}

The key to obtaining rapid solutions of large problems using BEM is to completely bypass the coefficient matrix computation. We have developed a new Direct Boundary Element Variational formulation that is used in conjunction with iterative solvers from the Krylov family, and a new Multilevel Fast Multipole Method (MLFMM) that facilitates extremely fast matrix-vector product computation. This approach overcomes the limitations of traditional BEM and allows us to perform fast NVH analysis of large problems in the midhigh frequency regime. We demonstrate the accuracy of our predictions by comparing with several closed form analytical solutions. Noise radiated by a gear box housing was analyzed as an example automotive $\mathrm{NVH}$ application.

\section{INTRODUCTION}

The Boundary Element Method (BEM) is widely used to predict the sound radiation from automotive components. BEM codes are popular since they involve only surface discretization and solve exterior (infinite domain) problems ( Figure 1) naturally. However, conventional $B E$ methods suffer from a major drawback the BEM coefficient matrices are fully populated and frequency dependent. This severely limits the size of models that can be built. The largest models that can be analyzed presently are limited to about 10,000 unknowns. The dimensions of the elements are related to the frequency range of interest, and thus a limita- tion on model size restricts the frequency range over which the BE model is useful. Presently, the usefulness of BEM is restricted to coarse models of small objects such as engine blocks, in the low frequency regime. In spite of its elegance and power, conventional BEM cannot be applied to aircraft interior, submarine exterior or architectural acoustics problems.

The Fast Multipole Method (FMM) is a recent breakthrough [1], which makes it possible to build intricate BEM models of real life systems and perform acoustic simulations efficiently. We have developed advanced computational tools based on BEM and FMM to facilitate simulation of very large sound-structure interaction problems. The following sections describe the research work performed.

\section{DIRECT BE FORMULATIONS}

COLLOCATION The Direct BEM formulation relates the acoustic pressure on the surface of the radiating body to the surface normal velocity through the free-space Greens function $G(P, Q)$.

$c(P) p(P)=\int_{\partial \Omega}\left(\frac{\partial p}{\partial n}(Q) G(P, Q)-p(Q) \frac{\partial G(P, Q)}{\partial n_{q}}\right) d \Omega$

where $\partial \Omega$ is the surface of the radiating object, $p$ is the sound pressure, $\partial p / \partial n$ is the normal derivative of pressure, and $P$ and $Q$ are points on the boundary $\partial \Omega, c(P)$ is proportional to the solid angle at point $P$. Pressure, velocity or impedance boundary conditions can be specified on different parts of the boundary.

Our MLFMM - Collocation BEM is similar to the one by Sakuma [2], with one crucial difference. Sakuma uses constant basis functions, meaning that the pressure and acoustic particle velocity do not vary over each triangular or quadrilateral patch. The resulting pressure field will have inter-element discontinuities and a large number of elements are required for an accurate solution. We allow higher order variable interpolation (linear, quadratic, cubic) in our boundary element formulations to reduce the number of unknown variables, and increase solution accuracy. 


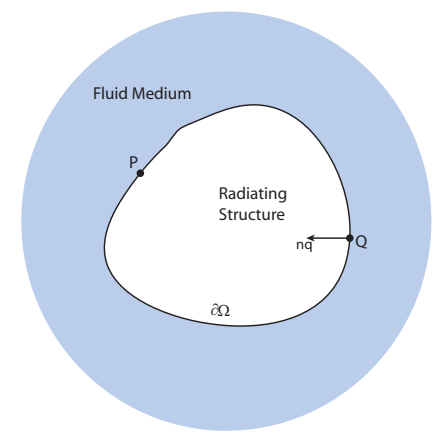

Figure 1: Problem domain for exterior sound radiation

VARIATIONAL FORMULATION In the variational formulation the problem is recast as a minimization problem. We use a combination of the Helmholtz integral equation and its normal derivative to construct a quadratic functional representing sound power. The functions that make the functional stationary automatically solve the integral equations on the specified surfaces. This formulation was originally developed by Pierce [3] for prescribed velocity boundary conditions on the surface. We have extended this to include all combinations of Dirichlet, Neumann and Robin boundary conditions on parts of the surface.

The functional for the case of prescribed velocity boundary conditions is given as

$$
\begin{aligned}
J_{v}[p]= & -\int_{S_{v}} \int_{S_{v}} p(p) \overline{p_{n}(q)} \frac{\partial G(p, q)}{\partial n_{p}} d s_{q} d s_{p} \\
& +\frac{1}{2} \int_{S_{v}} \int_{S_{v}} p(p) p(q) \frac{\partial^{2} G(p, q)}{\partial n_{p} \partial n_{q}} d s_{q} d s_{p} \\
& +\frac{1}{2} \int_{S_{v}} p(p) \frac{\overline{p_{n}(q)}}{2} d s_{p}
\end{aligned}
$$

One of the benefits of using a variational approach is that if we know of an approximate solution to the surface pressure $p$, from the physics of the problem, Equation 2 yields a very accurate expression for sound power - that is the error in sound power is $O\left(\epsilon^{2}\right)$, when the error in sound pressure is $O(\epsilon)$. During the iterative solution process, the solution for sound pressure is gradually refined. If the sound power is the quantity of interest, the iteration converges very rapidly.

\section{FAST MULTIPOLE METHOD}

In this section, we will give a brief overview of the The Fast Multipole Method (FMM), its application to the acoustic wave equation, and highlight how our innovations result in dramatic acceleration of FMM. FMM was originally developed by Barnes and Hut [4] for computing all pairwise interactions in the gravitational $n$-body problem. A rigorous mathematical framework for this approach was developed by Greengard [5].

FMM provides an efficient solution to the problem of computing the acoustic field caused by a cluster of $m$ sources, at $n$ observation locations. Figure 2 illustrates this idea for three sources and four observation points. Naive computation of all interactions requires $O(m n)$ calculations, in this case 12 calculations.

In the multipole method shown in Figure 2(b), the point sources $P_{i}$ inside the source cluster are combined to form a higher order source located at the cluster center $M$ (also called the multipole expansion center). The acoustic field at observation points sufficiently far from the source cluster can now be calculated as the field produced by a single multipole source located at $M$. If all the observation points $q_{j}$ are located near $L$ (called the local expansion center), the field at $L$ due to the multipole source at $M$ is first computed, and a Taylor series expansion is used to calculate the field at the each nearby observation point. The transfer function $T_{M L}$ between the source and observation clusters needs to be computed only once. Multipole method thus needs only $O(m+n)$ calculations for computing the field at all the observation points, a tremendous advantage as the number of sources and observation points increase.

The key to the FMM method is the expansion of the free space Green's function $G\left(P_{i}, q_{j}\right)$ between the source point $P_{i}$ and observation point $q_{j}$ in terms of propagating plane waves as shown in Equation 3.

$$
G\left(P_{i}, q_{j}\right)=\frac{i k}{16 \pi^{2}} \int_{S^{2}} E_{P_{i} M}(\hat{s}) T_{M L}(\hat{s}) E_{L q_{j}}(\hat{s}) d s
$$

In Equation 3, $\hat{s}=(\sin \theta \cos \phi, \sin \theta \sin \phi, \cos \theta)$ is a point on the unit sphere $S^{2}$, and represents the plane wave propagation direction, $E_{P_{i} M}(\hat{s})=\exp \left(i k \hat{s} \cdot \vec{r}_{P_{i} M}\right)$ is the radiation function, $T_{M L}$ is the transfer function in terms of Hankel functions [2], and $E_{L q_{j}}(\hat{s})=$ $\exp \left(i k \hat{s} \cdot \vec{r}_{L q_{j}}\right)$ is the local expansion function.

In the BEM framework, the acoustic sources and ob- 


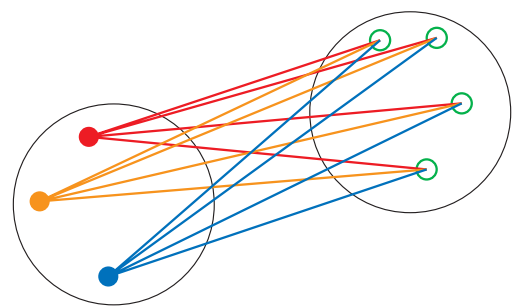

(a) Naive computation

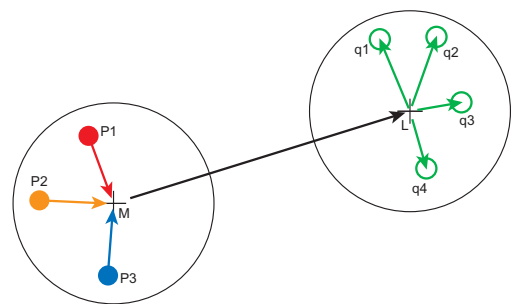

(b) Multipole computation

Figure 2: Concept of the multipole method. Solid circles represent sources and hollow circles represent observation points.

servation points are located on the surface mesh. In the collocation method, observation points are located at the nodes and the sources are located at the quadrature points on the elements, whereas in the variational method, both sources and observation points are located at the quadrature points. The product of the boundary element coefficient matrix $A$ with a vector $x$ is nothing but the acoustic field at the surface observation points due to surface sources whose strengths are determined by the vector $x$. This is exactly the calculation that FMM performs efficiently. Hence we use FMM to compute the BEM matrix vector products.

\section{MULTILEVEL FMM}

A cubical cell enclosing all the sources is taken as the root cell (Level 0 ). It is subdivided into eight children cells, each representing an octant of root cells domain to form Level 1. This process is continued in a recursive manner to create cells up to the desired number of levels, as illustrated in Figure 3 for two-dimensional problems. When sub-dividing a parent cell, if the child cell to be created does not contain any sources or observation points, it is not created. The cell length is denoted by $d$ and the cell diameter is $D=\sqrt{3} d$. The total number of levels $h$ is called the height of the tree.

At any level $l$, the cells that are adjacent to a given cell are called its neighbors. The interaction set for a given cell contains all the children of its parents neighbors. Among the cells that interact with a given cell, the neighbor cells are called the near cells, as they are too close for the multipole solution to be valid. The rest of the cells are designated as far cells. Any given cell can have a maximum of 27 near cells, and $6^{3}-3^{3}=189$ far cells. The near and far cell lists for all the cells are constructed recursively. Figure 3 shows the near and far lists for cells at each level.

MLFMM is comprised of the following steps.

Upward Pass: It starts at the level $h-1$ and does a bottom-up traversal of the cell tree till level 2. At level $h-1$ the multipole expansions of the individual sources within a cell are aggregated at the cell center. At higher levels, the child cell expansions are translated to the parent cell center. At the end of the upward pass, the multipole source strengths of all the cells in the hierarchy are known.

Downward Pass: It is a top-down traversal of the cell tree starting at level 2 and ending at level $h-1$. For a cell in level 2, the far field or the contributions of the far cells are computed by applying the transfer function to the multipole source strengths computed in the upward pass. In subsequent levels, the acoustic field at the parent cells center is translated to the child centers, to which the child's far field is added. On reaching level $h-1$, the far field at every cell center is known.

The main idea of MLFMM is clustering sources at various spatial lengths and computing interactions with far cells by means of multipole expansions. Near cell interactions are computed directly as illustrated in Figure 4.

We have made a number of proprietary enhancements to improve the accuracy and to speed up information transfer between levels in the upward and downward passes.

\section{VALIDATION AND PERFORMANCE SCALING}

OSCILLATING SPHERE EXAMPLE Solution accuracy and speed are the two important characteristics that we studied. To validate the accuracy for the FMM-BEM solutions, and to observe the scaling of analysis time and memory usage with problem size, we built a series of increasingly fine discretizations of a sphere. The mesh sizes range from 300 nodes to 250,000 nodes. The computed numerical solutions for the oscillating sphere problems are compared with exact analytical solution given by Equation 4, to verify solution accuracy. The simulations were performed on a $2 \mathrm{GHz}, 64$-bit AMD machine with $3 G B$ of physical RAM and running Linux oper- 


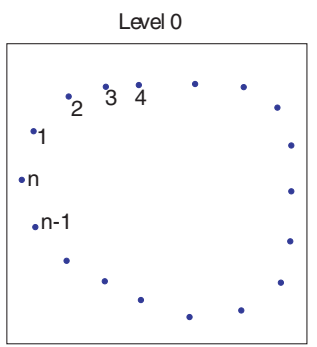

Level 3

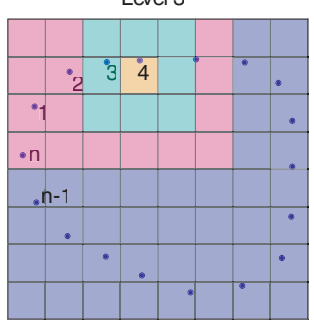

Figure 3: Computational cell hierarchy and construction of near and far lists. ating system. Both methods use iterative GMRES solver. The solution is deemed to have converged when the residual $r=\|A x-b\|_{2} /\|b\|_{2}$ is less than $10^{-5}$. FMM-BEM run-times and memory usage are compared with conventional BEM to demonstrate its superior performance.

For a sphere of radius $a$, oscillating in the z-direction with an unit amplitude, at angular frequency $\omega$ the analytical solution for sound pressure field is given by

$$
p(r, \theta, \phi)=3 i Z_{0} \cos \theta \frac{h_{1}(k r)}{h_{0}(k a)-h_{2}(k a)}
$$

where $h_{l}$ is the spherical Hankel function of order $l, Z_{o}$ is the characteristic impedance of the medium, and $k=\omega / c$ is the wave number.

Run Time Comparison Figure 5(a) plots the total run time for analyzing one frequency step as a function of mesh size given by the number of nodes. In FMM-BEM, the number of levels of the multilevel cell hierarchy is varied with mesh size to keep the near field computations to a minimum. Each different point marker on the FMM-BEM line represents a different tree height.

From Figure 5(a), it is apparent that FMM-BEM is much faster than conventional BEM for all mesh sizes and becomes more so with increasing mesh size. Run time for Conventional BEM scales as $O\left(N^{2}\right)$ while FMM-BEM scales as $O(N \log N)$. BE models containing about 5000 nodes are typical in the auto industry. For the oscillating sphere problem containing 5000 nodes, conventional BEM takes 831 seconds per frequency step while our FMM-BEM implemen- tation takes only 13 seconds, a factor of 64 speedup over current technology.

Memory Usage Peak memory usage is plotted in Figure 5(b). Memory requirements for FMM-BEM grow less rapidly than conventional BEM. For small models, conventional BEM needs less memory. The crossover between the two methods occurs at about 2000 nodes - for larger models it is advantageous to use FMM-BEM. For a typical industrial model containing 5000 nodes, conventional BEM needs $407 \mathrm{MB}$ while FMM-BEM needs only $151 \mathrm{MB}$, a factor of $3 \mathrm{im}-$ provement.

Increasing the number of FMM levels, reduces the number of near-field interactions that must be computed directly. A larger portion of the interactions will be in the far-field that is computed very efficiently using FMM. This reduces the memory requirements and further increases the solution speed. The jumps in Figure 5 are caused by increasing the number of FMM levels so that the terminal cell has approximately same the linear dimensions as the element size.

Solution Accuracy The following relative error norm is defined to measure the deviation of the computed numerical solutions from the exact analytical solution.

$$
e_{2}=\frac{\left\|p_{\text {numerical }}-p_{\text {exact }}\right\|_{2}}{\left\|p_{\text {exact }}\right\|_{2}}
$$

Both conventional BEM and FMM-BEM yield very accurate solutions, the relative error $e_{2}$ in the surface sound pressure is less than $0.6 \%$ as shown in Fig- 


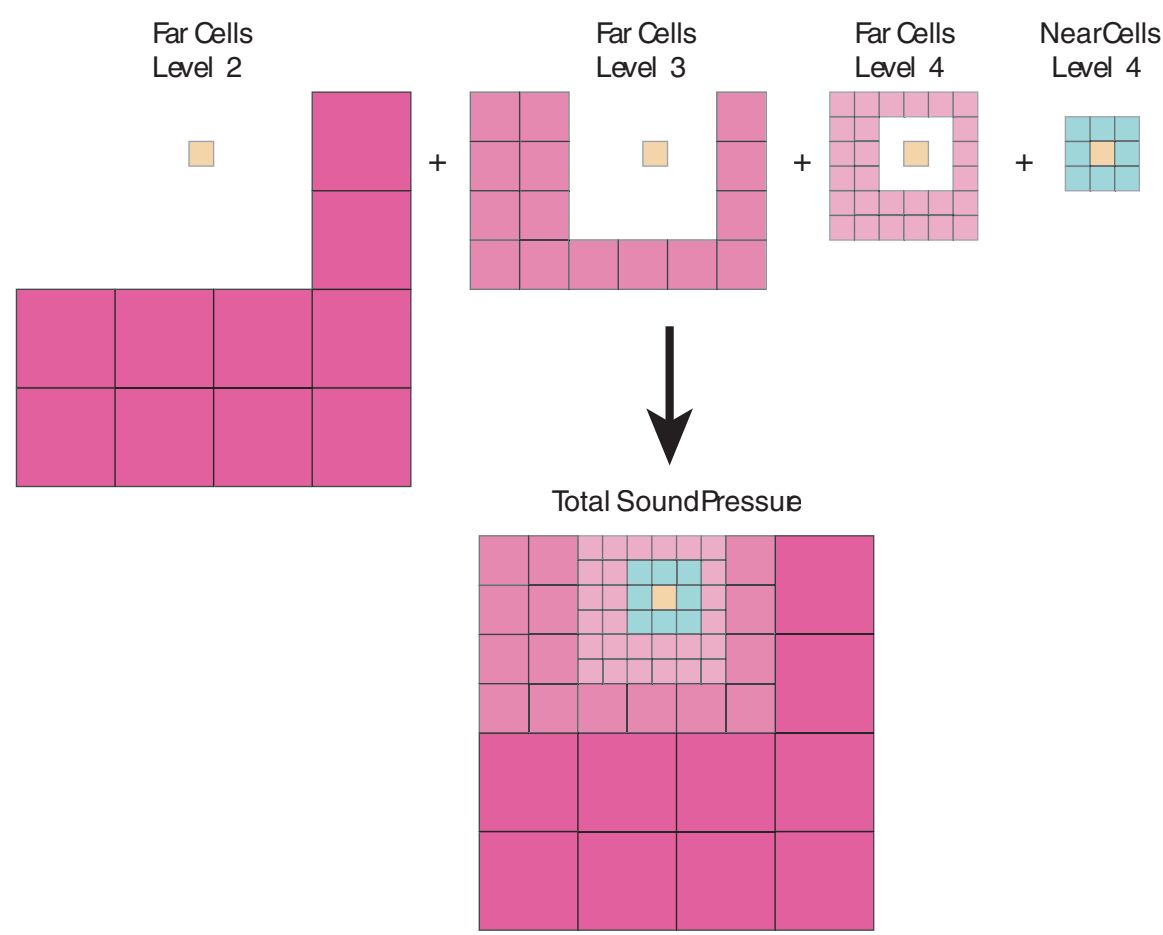

Figure 4: MLFMM concept - increasing cluster size with distance from the observation cell causes larger numbers of sources to be accumulated.

ure 6. For many engineering applications, $e_{2}<2 \%$ is acceptable.

\section{GEARBOX RADIATION}

Sound radiation from two automotive gearbox housings with an assumed velocity boundary condition was analyzed to demonstrate the application of our methods to automotive NVH problems.

TRANSFER CASE An automotive transfer case distributes the torque between the front and the rear wheels. Noise radiation from the transfer case housing is an important factor affecting product quality. Figure 7 shows the structural finite element mesh of the transfer case.

The structural finite element mesh provides the geometry input to sound field computation, while the structural vibration provides the excitation. The structural FE mesh is usually very fine as it needs to accurately model the geometry and capture stress concentrations. The radiating surface, obtained by skinning the structural FE mesh is too large to be used for acoustic radiation calculation by conventional BEM. A mesh coarsening process is used to reduce the number of nodes in the acoustic $B E$ mesh, while trying to retain the geometric features. Mesh coarsening process in- creases cycle time and cost of BE model preparation. The sole reason for mesh coarsening is to prepare a $\mathrm{BE}$ mesh that can be handled by conventional BEM. FMM-BEM does not have this mesh size limitation and completely eliminates the need for mesh coarsening step.

The BEM mesh considered for analysis was obtained by skinning the structure FM mesh. The BE Mesh has 44202 elements and 41288 nodes. The representative element length is about $7.8 \mathrm{~mm}$, and BE Model is valid till $7255 \mathrm{~Hz}$. In order to demonstrate the accuracy of the FMM-BEM solution for an arbitrary radiator geometry, an assumed surface velocity distribution induced by a point monopole source located inside the transfer case was chosen. This surface velocity was chosen by design, as it allows us to compare the FMM-BEM solution for the transfer case geometry with the exact analytical solution (pressure field due to a point source in free space). The sound pressure on the transfer case surface is shown in Figure 8 . The field point pressure at a point $1 \mathrm{~m}$ from the transfer case is shown in Figure 9. The FMM-BEM solution is in excellent agreement with the analytical solution.

TRANSMISSION HOUSING The ability to handle large intricate models, allows us to include very fine geometric features such as ribs which are important 


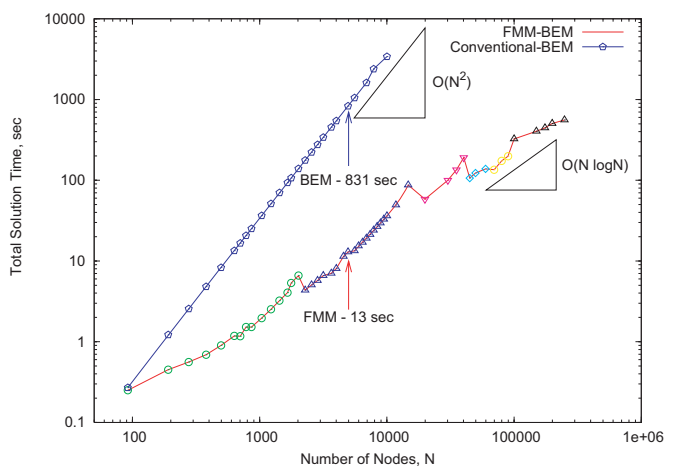

(a) Total solution time per frequency step.

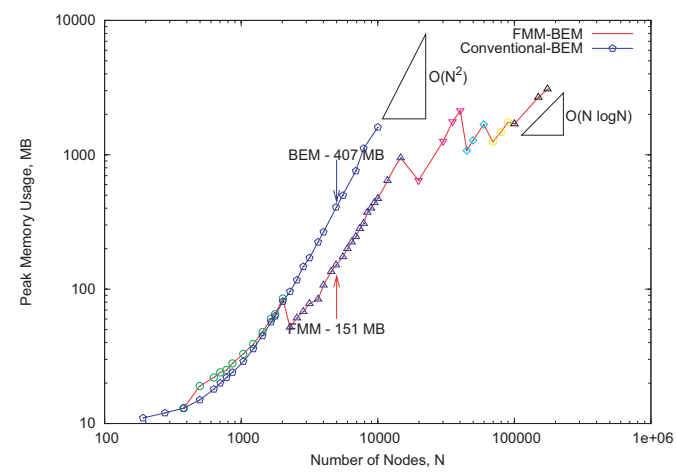

(b) Peak Memory Usage.

Figure 5: Performance comparison - FMM-BEM vs. conventional BEM.

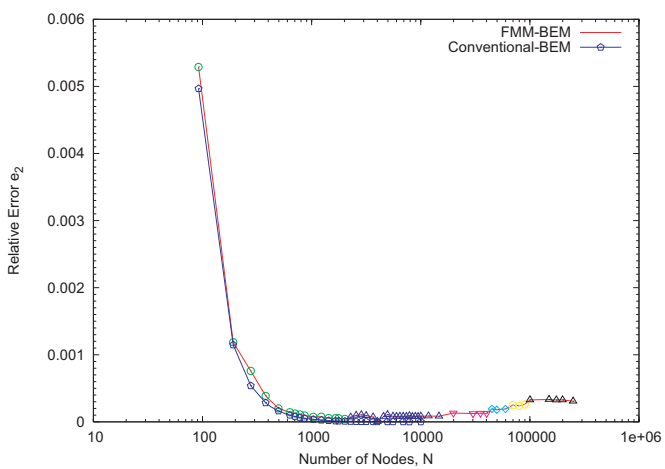

Figure 6: Solution Accuracy - FMM-BEM vs. conventional BEM.

for high frequency sound radiation. In order to push the envelope for model size, an acoustic BE model of a transmission housing model containing 422,548 nodes was built and analyzed. This is the largest model we have analyzed to date and is about 34 times larger than the models allowed by current technology. This model took 7.2 Hours of CPU time and 3.6 GB of total memory on a $2 \mathrm{GHz} 64$-bit AMD machine. The computer had only 3 GB of physical RAM. The speed can be further improved for this example by adding more RAM so that the use of virtual memory is minimized. A problem of this size simply could not have been solved by the BEM without use of the FMM technique. We are currently working on performance tuning and reducing the memory footprint.

\section{CONCLUSION}

MLFMM is a viable way of extending the applicability of BEM to very large problems and to higher frequencies. MLFMM results in impressive performance improvements while retaining the solution accuracy. We are currently working on efficient preconditioning techniques for faster convergence of the iterative solvers, on further reducing the memory usage, and performing analysis of automotive NVH problems such as the interior sound field in SUV cabins.

\section{REFERENCES}

[1] V. Rokhlin. Diagonal forms of translation operators for the Helmholtz equation in three dimensions. Applied and computational harmonic analysis, 1:82-93, 1993.

[2] T. Sakuma and Y. Yasuda. Fast multipole boundary element method for large-scale steady-state sound field analysis. part 1: setup and validation. Acta Acustica, 88:513-525, 2002.

[3] A. D. Pierce. Stationary variational expressions for radiated and scattered acoustic poer and related quantities. IEEE Journal of Oceanic Engineering, 12(2):404-411, April 1987.

[4] J. Barnes and P. Hut. A hierarchical $o(n \log n)$ force-calculation algorithm. Nature, 324(4):446449, December 1986.

[5] L. Greengard. The rapid evaluation of potential fields in particle systems. PhD thesis, Massachussets Institute of Technology, 1987. 


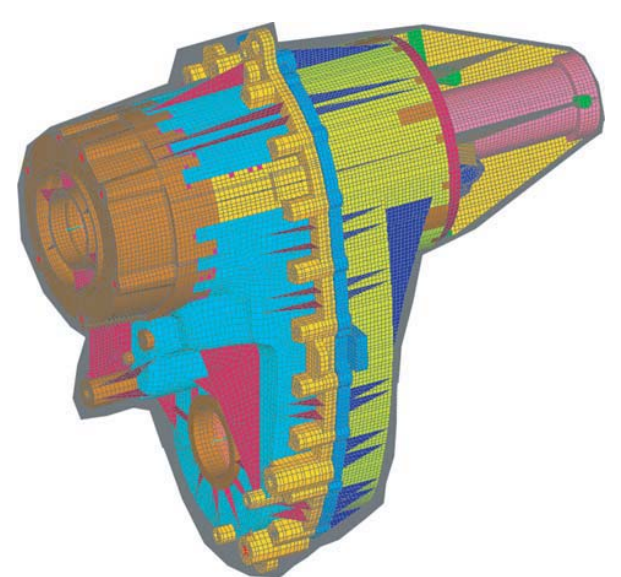

Figure 7: Transfer Case - Structural Mesh.

\section{ACKNOWLEDGMENTS}

This material is based upon work supported by the National Science Foundation under Grant No. 0548629. Any opinions, findings, and conclusions or recommendations expressed in this material are those of the author(s) and do not necessarily reflect the views of the National Science Foundation.

The authors thank Sue Stroope and Ravi Narayanaswamy from BorgWarner TorqTransfer Systems for providing the transfer case finite element model that was used as an example in this paper.

\section{CONTACT}

Rajendra Gunda, Ph.D

Phone: 614-559-3898

Email: rajendra.gunda@ansol.com

Web: http://www.ansol.com

\section{ABBREVIATIONS}

BEM Boundary Element Method

FMM Fast Multipole Method

MLFMM Multilevel Fast Multipole Method

NVH Noise, Vibration and Harshness

FEM Finite Element Method

GMRES Generalized Minimum Residual Method 


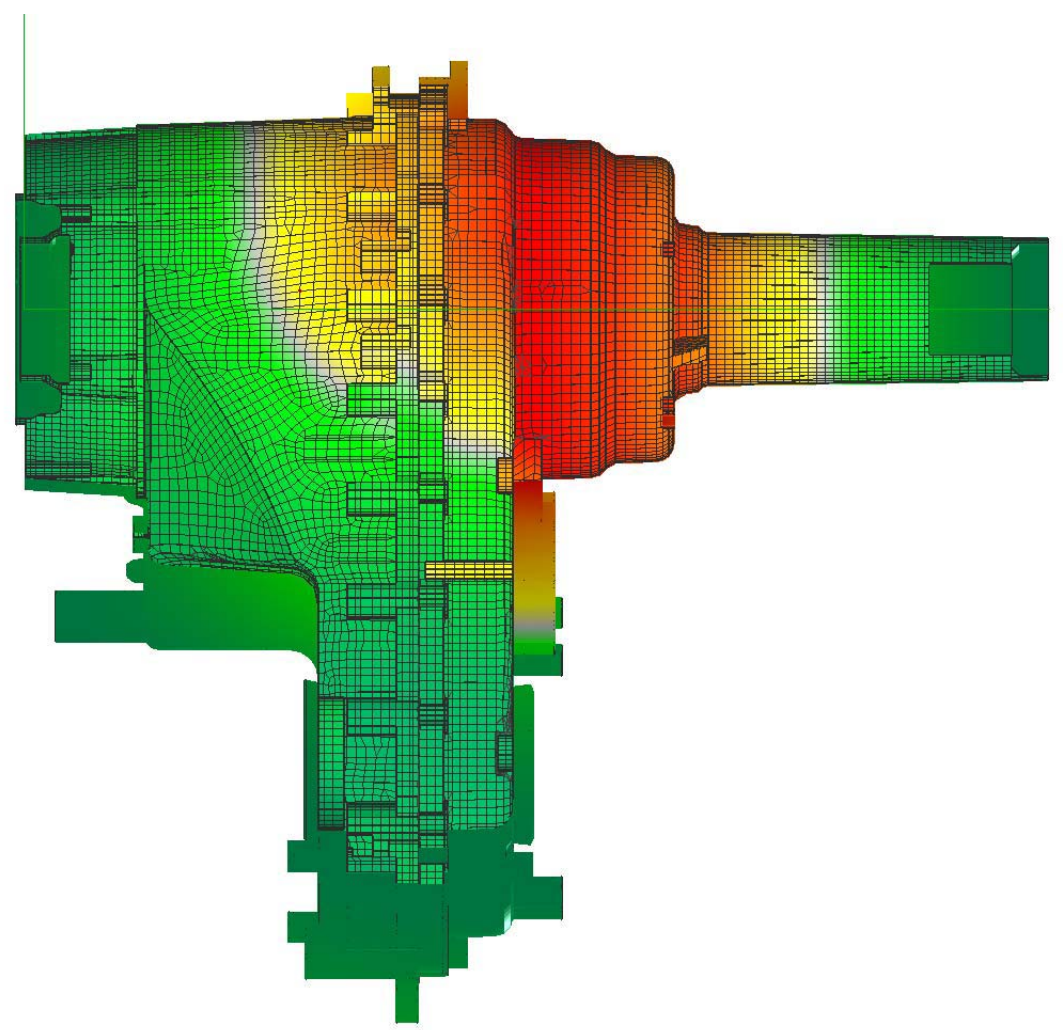

Figure 8: Sound pressure (real part) on the transfer case surface at $500 \mathrm{~Hz}$.

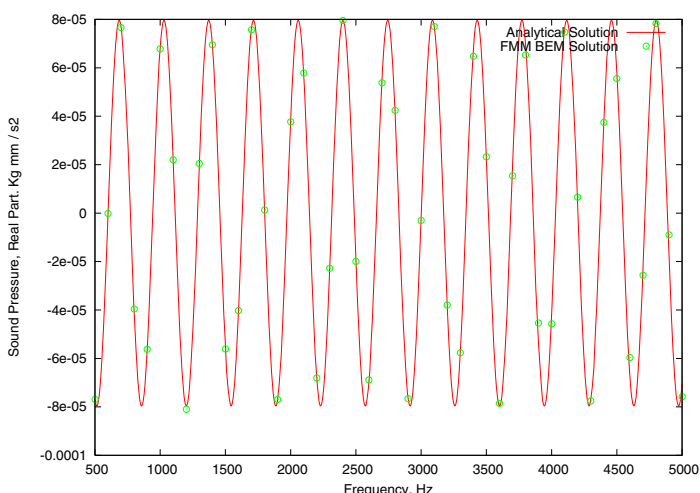

(a) Real Part

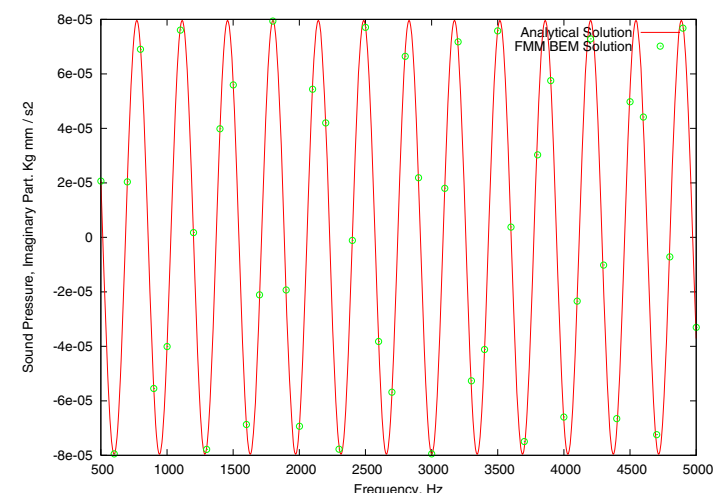

(b) Imagiequency, . Part

Figure 9: Sound pressure at a field point $1 \mathrm{~m}$ away from the transfer case. 